\title{
Investigation for the Colistin Resistance Genes $m c r-1$ and $m c r-2$ in Clinical Enterobacteriaceae Isolates from Cats and Dogs in Switzerland
}

\author{
Sabrina Simmen $^{\text {a }}$, Katrin Zurfluh ${ }^{\text {a }}$, Magdalena Nüesch-Inderbinen ${ }^{\text {a }}$, Sarah Schmitt ${ }^{\text {b }}$ \\ ${ }^{a}$ Institute for Food Safety and Hygiene, University of Zürich, Winterthurerstrasse Zürich, \\ Switzerland. \\ ${ }^{\mathrm{b}}$ Institute of Veterinary Bacteriology, Vetsuisse Faculty, University of Zürich, Switzerland
}

\begin{abstract}
Colistin resistant Enterobacteriaceae have emerged in humans, food producing animals and food. The aim of this study was to assess the occurrence of MCR-1 and MCR-2 producers among Enterobacteriaceae isolated from companion animals admitted to the University of Zürich veterinary clinic. A total of 347 isolates (231 from dogs, 116 from cats) were analysed. Thereof, 274 (79\%) were from urine and 73 (21\%) from surgical sites, abscesses and other sources. All isolates were screened by polymerase chain reaction (PCR) for the presence of the plasmid-mediated colistin resistance genes mcr-land mcr-2. Phenotypic colistin resistance was screened for by the antibiotic broth microdilution method. None of the isolates tested positive by PCR for the mcr-1 or the mcr-2 gene. Broth microdilution tests revealed MICs of $2 \mathrm{mg} / \mathrm{L}-64 \mathrm{mg} / \mathrm{L}$ of colistin for four isolates (two Enterobacter spp., one Klebsiella spp. and one E. coli) from dogs (one from skin exudate, one from a surgical site and two isolates from urine). This study documents the presence of non-transmissible colistin resistance in clinical isolates from dogs and cats and confirms that so far, companion animals in Switzerland do not represent a reservoir for plasmid-mediated colistin resistant Enterobacteriaceae.
\end{abstract}

Keywords: Colistin; resistance; Escherichia coli; companion animals

\author{
Abbreviations \\ polymerase chain reaction (PCR) \\ lipopolysaccharides (LPS) \\ multidrug resistant (MDR) \\ Escherichia coli (E. coli) \\ Klebsiella pneumoniae (K. pneumoniae) \\ Enterobacter cloacae (E. cloacae) \\ European committee on antimicrobial susceptibility testing (EUCAST) \\ minimal inhibitory concentration (MIC) \\ 4-amino-4-deoxy-L-arabinose (L-Ara4N) \\ phosphoethanolamine (PEtN)
}

\section{INTRODUCTION}

Polymyxins are cationic polypeptide antibiotics that interact with the lipopolysaccharides (LPS) and phospholipids in the outer membrane of Gram-negative bacteria. In veterinary medicine, colistin (polymyxin E) is used regularly for the treatment of gastrointestinal infections in livestock, whereas polymyxin B is topically applied for the treatment of otitis media and skin infections in small animals [1]. In human medicine, colistin has become one of the last-resort antimicrobials for treating lifethreatening infections caused by multidrug resistant (MDR) bacteria, and the emergence of plasmidmediated polymyxin resistance in Enterobacteriaceae severely compromises its use [2]. Since its first 
description in China in 2015 [3], the colistin resistance gene $m c r-1$ has been reported worldwide in isolates from humans, food producing animals and food [4]. Furthermore, a novel variant, $m c r-2$ was detected recently in livestock associated E. coli in Belgium [5].

A recent report from China shows that MCR-1 producing E. coli can colonize companion animals available in pet markets and can be transferred between companion animals and humans [6]. Hence, in addition to food animals and humans, companion animals can represent a reservoir of colistinresistant E. coli. The aim of this study was to assess the prevalence rate of MCR-1 and MCR-2 producing Enterobacteriaceae in clinical strains obtained from companion animals admitted to the small animal veterinary clinic of the University of Zürich, Switzerland between February 2012 and July 2016. In addition, phenotypic resistance to colistin among the isolates was determined.

\section{Materials AND Methods}

\subsection{Strain Collection}

A collection of 347 isolates recovered between 2012 and 2016 from 231 dogs and 116 cats presented to the University of Zürich veterinary clinic was available for analysis.

The isolates were obtained from urine $(n=274)$, surgical sites $(n=39)$, abscesses and wounds $(n=27)$, and other sites $(\mathrm{n}=7)$. The isolates included E. coli $(\mathrm{n}=254)$, Klebsiella pneumoniae $(\mathrm{n}=32)$, Enterobacter cloacae $(\mathrm{n}=29)$, Proteus mirabilis $(\mathrm{n}=9)$, Klebsiellaspp. $(\mathrm{n}=8)$, Proteus spp. $(\mathrm{n}=8)$, Enterobacteraerogenes $(\mathrm{n}=2)$, Proteus vulgaris $(\mathrm{n}=2)$, Citrobacter freundii $(\mathrm{n}=1)$, Citrobacter koseri $(\mathrm{n}=1)$ and Enterobacter $\mathrm{sp}$. $(\mathrm{n}=1)$.

\subsection{Screening for $m c r-1$ and $m c r-2$ by PCR}

DNA was extracted using a standard heat lysis protocol. All isolates were screened for the $m c r-1$ and mcr-2 genes using primers (custom synthesized by Microsynth, Balgach, Switzerland) and PCR conditions described previously [3,7]. Positive controls were included.

\subsection{Antimicrobial Susceptibility Testing}

Phenotypic colistin resistance was screened for using the broth microdilution method and a single selected screening concentration of $2 \mathrm{mg} / \mathrm{L}$ of colistin, which represents the susceptibility breakpoint according to the guidelines of the European committee on antimicrobial susceptibility testing, EUCAST (www.eucast.org). Screening was applied to all isolates except Proteus spp., which possess intrinsic resistance to polymyxins. Isolates with growth in the screening medium were further tested for susceptibility to colistin by broth microdilution, and minimal inhibitory concentrations (MIC) were determined as described by EUCAST (www.eucast.org).

\section{RESUlTS AND DisCUSSION}

PCR results for all isolates remained negative. Based on this finding, no evidence for the occurrence of MCR-producing Enterobacteriaceae in companion animals in Switzerland need be established.

Screening for phenotypic resistance to colistin gave rise to seven isolates (2\% of all isolates, all from dogs). Thereof, four isolates were resistant to colistin upon susceptibility testing by broth microdilution according to EUCAST. All four isolates originated from dogs $(1.7 \%$ of the isolates from dogs): one E. cloacae (MIC $64 \mathrm{mg} / \mathrm{L}$ ) isolated from a skin exudate, one Enterobacter spp. (MIC $64 \mathrm{mg} / \mathrm{L}$ ) from a surgical site (abdominal cavity), one Klebsiella spp. (MIC $64 \mathrm{mg} / \mathrm{L}$ ) isolated from urine and one E. coli (MIC $2 \mathrm{mg} / \mathrm{L}$ ) isolated from urine. While non-transmissible polymyxin resistance has been described for a number Gram-negative species, clinical $E$. coli isolates with this resistance mechanism are reported rarely [8,9], hence, the isolation of polymyxin resistant $E$. coli from a dog with UTI warrants attention. Although the mechanisms leading to colistin resistance were not investigated in this study, numerous complex mechanisms, predominantly those involving modifications of the outer cell membranes have been documented, including alterations of lipid A with 4-amino-4-deoxy-L-arabinose (L-Ara4N) and phosphoethanolamine (PEtN) [10]. The activation of such mechanisms is triggered by environmental stimuli and the development of polymyxin resistance under therapy with colistin has been observed in several instances of human infections with Klebsiella spp. [11,12]. Thus, it cannot be excluded that the occurrence of the colistin resistant 
isolates observed in this study is associated with polymyxin exposure. To avoid conditions that could promote the emergence of plasmid-mediated colistin resistance, judicious use of polymyxins in animals is of utmost importance.

Currently, the prevalence of MCR-producing enterobacterial clinical isolates from humans worldwide and in Switzerland is below 1\% [13]. However, recent studies indicate a rising trend and point to an ongoing transmission of colistin resistance determinants and/or colistin resistant $E$. coli isolates from animals to humans [2]. Identification of reservoirs and transmission risks are warranted in order to prevent the spread of MCR producers. Strategies are needed to maintain the low prevalence of MCR producers in humans as well as animals, including screening of isolates from different sources.

\section{Conclusion}

This study represents the first screening for $m c r$ genes in clinical isolates from companion animals in Switzerland and contributes to a better understanding of the epidemiology of $\mathrm{mcr}$ genes and the identification of risks to human and animal health. Although so far, dogs and cats in Switzerland do not represent a reservoir for plasmid-mediated colistin resistant Enterobacteriaceae, contact with companion animals from countries with a high prevalence of antimicrobial resistance, e.g. through international trade with pets or through travel, may be considered a risk with regard to the possible transmission of MCR producing bacteria.

\section{ACKNOWLEDGEMENTS}

This work was partly supported by the Swiss Federal Office of Public Health, Division Communicable Diseases.

\section{REFERENCES}

[1] Catry, B., Cavaleri, M., Baptiste, K., Grave, K., Grein, K., Holm, A., Jukes, H., Liebana, E., Lopez Navas, A., Mackay, D. Magiorakos AP, Moreno Romo MA, Moulin G, Muñoz Madero C, Matias Ferreira Pomba MC, Powell M, Pyörälä S, Rantala M, Ružauskas M, Sanders P, Teale C, Threlfall EJ, Törneke K, van Duijkeren E \& Torren Edo J. Use of colistin-containing products within the European Union and European Economic Area (EU/EEA): development of resistance in animals and possible impact on human and animal health. Int. J. Antimicrob. Agents 46, $297-$ 306 (2015).

[2] Schwarz, S., Johnson, A. P. Transferable resistance to colistin: a new but old threat. J. Antimicrob. Chemother. 71, 2066-2070 (2016).

[3] Liu, Y. Y., Wang, Y., Walsh, T. R., Yi, L. X., Zhang, R., Spencer, J., Doi, Y, Tian, G., Dong, B., Huang, X., et al. Emergence of plasmid-mediated colistin resistance mechanism MCR-1 in animals and human beings in China: a microbiological and molecular biological study. Lancet Infect. Dis. 16, 161-168 (2016).

[4] Poirel, L., Nordmann, P. Emerging plasmid-encoded colistin resistance: the animal world as the culprit. J. Antimicrob. Chemother. 71, 2326-2327 (2016).

[5] Xavier, B. B., Lammens, C., Ruhal, R., Kumar-Singh, S., Butaye, P., Goossens, H., MalhotraKumar, S. Identification of a novel plasmid-mediated colistin-resistance gene, $m c r-2$, in Escherichia coli, Belgium, June 2016. Euro. Surveill. 21, pii=30280 (2016).

[6] Zhang, X-F., Doi Y., Huang X., Li H-Y., Zhong L-L., Zeng K-J., Zhang, Y-F., Patil, S., Tian, GB. Possible transmission of $m c r-1$-harboring Escherichia coli between companion animals and human. Emerg. Infect. Dis. 22, 1679-1681 (2016).

[7] Liassine, N., Assouvie, L., Descombes, M. C., Tendon, V. D., Kieffer, N., Poirel, L., Nordmann, P. Very low prevalence of MCR-1/MCR-2 plasmid-mediated colistin resistance in urinary tract Enterobacteriaceae in Switzerland. Int. J. Infect. Dis. 51, 4-5 (2016).

[8] Gales, A. C., Jones, R. N., Sader, H. S. Global assessment of the antimicrobial activity of polymyxin B against 54731 clinical isolates of Gram-negative bacilli: report from the SENTRY antimicrobial surveillance programme (2001-2004). Clin. Microbiol. Infect. 12, 315-321 (2006).

[9] Urban, C., Tiruvury, H., Mariano, N., Colon-Urban, R., Rahal, J. J. Polymyxin-resistant clinical isolates of Escherichia coli. Antimicrob. Agents Chemother. 55, 388-389 (2011). 
[10] Olaitan, A. O., Morand, S., Rolain, J. M.Mechanisms of polymyxin resistance: acquired and intrinsic resistance in bacteria. Front. Microbiol. 5, 643 (2014).

[11] Lee, J., Patel, G., Huprikar, S., Calfee, D. P., Jenkins, S. G. Decreased susceptibility to polymyxin B during treatment for carbapenem-resistant Klebsiella pneumoniae infection. J. Clin. Microbiol. 47, 1611-1612 (2009).

[12] Bogdanovich, T., Adams-Haduch, J. M., Tian, G.-B., Nguyen, M. H., Kwak, E. J., Muto, C. A., Doi, Y. Colistin-resistant, Klebsiella pneumoniae carbapenemase (KPC)-producing Klebsiella pneumoniae belonging to the international epidemic clone ST258. Clin. Infect. Dis. 53, 373-376 (2011).

[13] Castanheira, M., Griffin, M. A., Deshpande, L. M., Mendes, R. E., Jones, R. N., Flamm, R. K. Detection of $m c r-1$ among Escherichia coli clinical isolates collected worldwide as part of the SENTRY Antimicrobial Surveillance Program in 2014 and 2015. Antimicrob. Agents Chemother. 60, 5623-562 (2016). 